\section{How do enzymes} work?

M.I. Page

The Bioorganic Chemistry of Enzymatic Catalysis.

Bergeron and Makoto Komiyama.

Wiley: 1984. Pp.312. £50.05, \$39.50.

IT is fashionable to justify the publication of work on some aspects of chemistry by invoking its relevance to the phenomena of life. Often this is unnecessary, because the chemistry is of interest in its own right (while sometimes the claim is exaggerated). In particular the no-man's-land between traditional disciplines is always exciting because this is where fresh understanding often emerges. The pioneering research of Myron Bender is a case in point; Bender helped to bridge the gap between chemistry and biochemistry and to define a new borderline area. Today the application of the techniques and phraseology of physical organic chemistry to enzyme catalysis is accepted practice and owes much to his work.

Our response to fundamental questions of life is usually to resort to models we think we understand. By definition, the model is a simpler system and only reflects a small part of the observed behaviour of the living system. Ill-defined separation of causes and effects in vivo may lead to only partial answers. termined by the question - in this case, how do enzymes work? In their book, Bender, Bergeron and Komiyama have
By Myron L. Bender, Raymond J.

The fullness of an answer is of course de-

aimed to show how an understanding of organic reaction mechanisms is relevant to an understanding of how enzymes work. They accept and propagate the view that the mechanisms - that is, the routes of bond making and breaking processes - of reactions are similar in non-enzyme and in enzyme-catalysed reactions. This is almost certainly correct. However, the number of amino acid residues of the enzyme directly involved in bond making and breaking is only a tiny percentage of the total number in the whole enzyme. What is the function of the rest of the enzyme's structure? Why can't the one or two amino acid residues involved in the "mechanism" catalyse the reaction as free amino acids? Besides enhancing the rate of reactions, enzymes are generally very discriminative in their choice of substrates. Why can't an enzyme catalyse the reaction of a compound which contains the necessary reactive function but is smaller than the normal substrate? Questions such as these are not addressed in the book.

It is not obvious that three authors have been involved in the writing, and the style throughout is uniform. This is an authoritatively written and well illustrated account of physical organic chemistry which should be useful to advanced undergraduate and graduate students wanting an understanding of the mechanism of reactions and an answer to why and how catalysis occurs. It is at its best when simple chemical reactions are discussed, but sadly fails to hint at the unsolved or controversial aspects of enzymatic catalysis.

M.I. Page is Professor and Head of the Department of Chemical and Physical Sciences at Huddersfield Polytechnic.

\section{Viruses in disease}

\section{Clive Sweet}

Concepts in Viral Pathogenesis.

Edited by Abner Louis Notkins and

Michael B.A. Oldstone.

Springer-Verlag: 1984. Pp.409. \$34.40,

DM 94.

Viral Pathogenesis and Immunology.

By Cedric A. Mims and David O. White.

Blackwell Scientific: 1984. Pp.398. Pbk

f14.80, \$26.

UNTIL recently, most virologists have shied away from studies of pathogenesis, regarding them as too difficult, less interesting or less satisfactory than the more molecular aspects of virology. Much attention has been given to the structure and biochemistry of viruses, and mechanisms of replication in cell culture, but virus pathogenicity - that is, the ways in which they produce disease - has been relatively neglected. This emphasis is changing and there is now a keen interest in the mechan- isms of disease production, possibly originating from a clearer understanding of host defence mechanisms but given impetus by the merging of interests of molecular virologists and those more concerned with the causation of disease. Pioneering work with segmented RNA viruses (reoviruses and orthomyxoviruses) has led to identification of virus genes and their products and has resulted in a better understanding of virus virulence at the genetic and molecular level. The new area of recombinant DNA technology has allowed these approaches to be extended to non-segmented RNA (polio) as well as DNA (herpes simplex, pseudorabies) viruses.

While studies on virus pathogenicity hold great promise, they demand a breadth of understanding across many specialized areas. Knowledge of virus structure, genetics and replication, together with broader aspects of cell and host biology, is required for a full appreciation of the subject. Consequently, the writing of a textbook on the subject is a daunting task. The two books reviewed here have tackled this problem in different ways.
The editors of Concepts in Viral Pathogenesis have chosen the multiauthor approach. No fewer than 75 authors have contributed a total of 53 articles under the headings of "Host Control of Viral Spread", "Host Range and Tissue Tropism", "Virus Maturation and Diversity", "Virus Persistence", "Virus-interaction with the Immune System", "Evolving Concepts in Viral Pathogenesis" and finally "Control of Viral Disease". The book is intended to be a series of minireviews and editorials, 1,000-2,000 words in length, each a "pithy distillation of the state-of-the-art".

Sadly, the book does not attain these objectives. Although all of the articles contain much information they often amount only to thumbnail sketches and are too short to be really useful. However, they are generally well referenced (with one notable exception where no references are given), allowing easy access to more detailed information. Inevitably, there is some duplication between chapters, (fortunately never contradictory), and some areas are ignored. For example, there is no chapter on virus-dependent damage (cytotoxicity) and the impression gained from reading the book is that viruses do not in themselves cause disease, this being almost entirely due to aberrations in host responses. A consideration in relevant chapters of how viruses avoid, counteract or fail to induce host responses would also have been useful. Nevertheless, the book is a helpful source of reference and concepts. Typographical errors are few but the occasional use of abbreviations without definition is annoying.

In contrast Mims and White have produced an excellent text with just two contributors. Their book is packed full of information in an easily digestible, interesting and entertaining style. It covers in considerable detail the various aspects of virus pathogenesis - basic characteristics of viruses, infection and spread of viruses through the body, the immune response to viral infection, mechanisms of disease production, determinants of viral virulence and host resistance, persistent infections and finally immunization against virus diseases - from the point of view of both the host and the pathogen.

The book is especially strong in its treatment of the immune response. Indeed, the depth of coverage of all topics makes it suitable not only for medical and science undergraduates and postgraduates but also for scientists in, or moving into, viral pathogenesis research, for whom the upto-date and extensive reference section should prove especially valuable. Clear diagrams and tables illustrate many points throughout the book and each chapter concludes with a summary and selected readings.

Clive Sweet is a Research Fellow in the Department of Microbiology, University of Birmingham. 